\title{
PERFIL EPIDEMIOLÓGICO DE TRAUMA ABDOMINAL SUBMETIDO À LAPAROTOMIA EXPLORADORA
}

\author{
Epidemiologic profile of abdominal trauma submitted to laporotomy \\ Nicolau Fernandes KRUEL, Valdriana Leandro de OLIVEIRA, Vanúzia Leandro de OLIVEIRA, \\ Ricardo de Deus HONORATO, Bianca Di PINATTI, Fábio Rosseto LEÃO
}

ABCDDV/538

Kruel NF, Oliveira VL, Oliveira VL, Honorato RD, Pinatti BD, Leão FR. Perfil epidemiológico de trauma abdominal submetido à laparotomia exploradora. ABCD Arq Bras Cir Dig 2007; 20(2):106-10.

RESUMO - Racional - No Brasil, no ano de 2004, a mortalidade por causas externas representaram 12,4\% do total de óbitos, ou seja, 124.470 óbitos O trauma abdominal está presente entre $13 \%$ e $15 \%$ de todos os acidentes fatais e contribui para mortes tardias por sepse. Objetivo - Descrever o perfil epidemiológico dos indivíduos com trauma abdominal que foram submetidos à laparotomia exploradora no período de 2003 a 2006 . Métodos - Estudo com delineamento transversal, descritivo e retrospectivo. A base para a coleta de informações foi de 185 indivíduos referentes a todos os traumas abdominais, submetidos à laparotomia exploradora, no período de 2003 a 2006. As variáveis estudadas foram: gênero, idade, meses do ano, causas de trauma, estruturas afetadas, tempo de internação, evolução, causas de óbito, traumatismo associados. Resultados - O gênero prevalente é masculino, com 87\%; a faixa etária mais freqüente foi a de 20 a 29 anos, com $44,3 \%$ do total; o ferimento por arma de fogo foi a principal causa, representando $51,9 \%$; a causa de óbito mais freqüente foi choque hipovolêmico, $47 \%$; das lesões extra-abdominais associadas a que mais prevaleceu foi o trauma torácico com $22,2 \%$. Conclusão - O sexo que predominou foi o masculino; a faixa etária predominante foi a terceira década; a causa principal foi o ferimento por arma de fogo; os órgãos mais afetados foram o intestino delgado, fígado e baço.

DESCRITORES - Trauma. Abdôme. Laparotomia.

\section{INTRODUÇÃO}

Segundo as concepções médico-sanitaristas, o trauma caracteriza-se pela existência de lesão, ferida, dano ou prejuízo produzidos no organismo e na mente. Seus condicionantes e causas são variados e predominantemente de origem externas, ainda que a expressão sintomática e as manifestações clínicas sejam influenciadas por mecanismos de funcionamento interno ao próprio organismo. Sabese que as suas conseqüências mórbidas não são totalmente previsíveis sendo, assim, limitadas as possibilidades de controle, prevenção, cura e reabilitação ${ }^{1}$.

No Brasil, no ano de 2004, a mortalidade por causas externas representou $12,4 \%$ do total de óbitos, ou seja, 124.470 óbitos $^{2}$. O impacto destas mortes pode ser analisado por meio do indicador relativo a "Anos Potenciais de Vida Perdidos" (APVP). Por incidirem com elevada freqüência no grupo de adolescentes e adultos jovens, os acidentes e as violências são responsáveis pelo maior APVP. No Brasil entre 1981 e 1991 este indicador aumentou $30 \%$ em relação a outros tipos de acidentes, enquanto que para as causas naturais, os dados encontrava-se em queda $^{3}$. O trauma das vísceras abdominais está presente entre $13 \%$ e $15 \%$ de todos os acidentes fatais e, não sur-

Trabalho realizado no Departamento de Cirurgia do Hospital Regional de São José - Homero Miranda Gomes, Florianópolis, SC, Brasil.

Endereço para correspondência: Nicolau Fernandes Kruel, e-mail: nfkruel@hotmail.com preendentemente, contribui de forma significativa às mortes tardias por sepse ${ }^{4}$.

A avaliação do abdôme é um dos componentes mais desafiadores da análise clínica inicial do traumatizado 5 . O trauma abdominal é uma fonte de morbimortalidade significativa e seu diagnóstico rápido é essencial com o intuito de minimizar a gravidade. As lesões penetrantes são mais diretas em sua apresentação e o diagnóstico mais fácil. A própria lesão penetrante chama a atenção por sua alta probabilidade de lesão intra-abdominal. $\mathrm{O}$ trauma abdominal fechado, por outro lado ocorre, usualmente em associação com lesão multisistêmica, fazendo com que seu diagnóstico seja mais complexo e desafiador ${ }^{6}$.

Os sinais peritoniais podem ser sutis e freqüentemente não valorizados devido à presença de dor maior em outros lugares, somados ao freqüente consumo de álcool, tóxicos, traumatismos encefálicos e raquimedular que acometem nesses pacientes. Aproximadamente um terço deles requerem laparotomia de urgência ${ }^{4}$.

A importância do traumatismo abdominal se deve ao fato de levar as vítimas mais graves a óbito na fase inicial do atendimento devido a hemorragias não controladas. Entretanto, a maioria destes traumas não acarreta risco de vida imediato ${ }^{7}$.

O objetivo deste estudo foi coletar dados epidemiológicos e dimensionar os fatores envolvidos no trauma abdominal que levaram à laparotomia exploradora. 


\section{MÉTODOS}

A presente investigação constitui-se de um estudo com delineamento transversal, descritivo e retrospectivo, tendo como base a coleta de informações referentes a todos os traumas abdominais, que tiveram como tratamento a laparotomia exploradora, ocorrido entre o período de 1/1/2003 a 31/12/2006. A coleta de dados foi realizada somente pelos autores do trabalho com preenchimento de dados a partir de protocolo desenvolvido no Departamento de Cirurgia do Hospital Regional de São José - Homero Miranda Gomes, incluindo as variáveis de interesse.

Foram utilizados como fonte de coleta de dados todos os prontuários médicos do referido hospital que se encontraram Serviço de Arquivamento Médico e Estatística. Foram analisados 185 prontuários de pacientes que sofreram trauma abdominal e submetidos à laparotomia exploradora.

As variáveis incluídas neste estudo foram: gênero, idade, meses do ano, causas, estruturas afetadas, evolução, tempo de internação e lesões extra-abdominais associadas. Para descrever as variáveis categóricas, foram calculadas suas freqüências absolutas (n) e relativas (\%) e seus respectivos intervalos de confiança de $95 \%$. As diferenças entre as categorias foram analisadas através do teste de diferença entre proporções. Foram consideradas significativas as diferenças quando o valor de $P \leq 0,05$. As análises foram realizadas através dos aplicativos Microsoft Excel e Arcus. Este estudo foi aprovado pela Comissão de Ética e Pesquisa do hospital.

\section{RESULTADOS}

Dos 185 prontuários de pacientes submetidos à laparotomia exploradora, $87 \%$ (161) eram do sexo masculino e $13 \%$ (24) do feminino.

A idade variou de 14 a 67 anos, e a média foi de 28,6 anos. A faixa etária mais acometida foi a de entre 20 e 29 anos com 44,3\% (82) do total, seguido pelo intervalo de 30 a 39 anos com 19,4\% (36) dos casos (Figura 1).

\section{Faixas etárias}

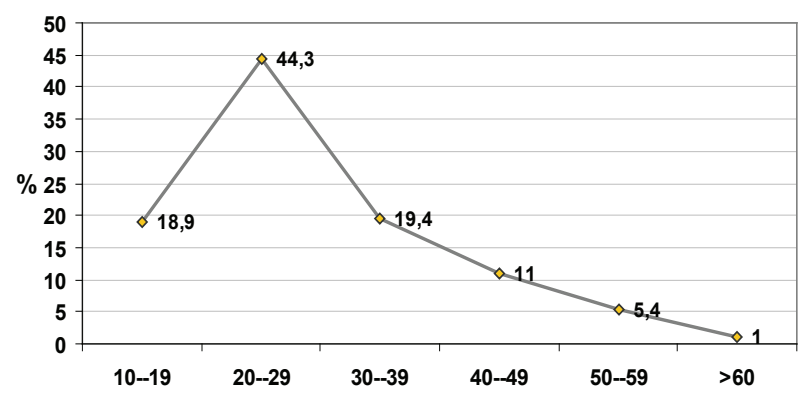

FIGURA 1 - Percentual de pacientes vítimas de traumas abdominais que foram submetidos à laparotomia exploradora, segundo a faixa etária. $P<0,05$

A distribuição entre os meses do ano revelou prevalência nos meses de outubro e dezembro.
O ferimento por arma de fogo foi a principal causa de trauma abdominal nos pacientes submetidos à laparotomia, representando $51,9 \%$ (96), seguido pelos ferimentos por arma branca com 16,7\% (31) (Tabelas 1 e 2) (Figura 2).

A Tabela 1 demonstra que a estrutura mais acometida foi o intestino delgado em $29,2 \%$ (54) dos pacientes, seguido pelo fígado em $25,4 \%$ (47).

TABELA 1 - Prevalência de lesão de órgãos vistos na laparotomia com base nos mecanismos de lesão. $P=0,0001$

\begin{tabular}{lcccc}
\hline \multirow{2}{*}{ Estruturas afetadas } & \multicolumn{2}{c}{ Contuso } & \multicolumn{2}{c}{ Penetrante } \\
\cline { 2 - 5 } & $\mathbf{n}$ & $\mathbf{\%}$ & $\mathbf{n}$ & $\mathbf{\%}$ \\
\hline Intestino delgado & 12 & $21,4 \%$ & 42 & $32,5 \%$ \\
Fígado & 10 & $17,8 \%$ & 37 & $28,7 \%$ \\
Baço & 25 & $44,6 \%$ & 17 & $13,2 \%$ \\
Intestino grosso & 7 & $12,5 \%$ & 35 & $27,1 \%$ \\
Estômago & 3 & $5,3 \%$ & 30 & $23,2 \%$ \\
Diafragma & 2 & $3,5 \%$ & 21 & $16,2 \%$ \\
Rim & 2 & $3,5 \%$ & 19 & $14,7 \%$ \\
Mesentério & 6 & $10,7 \%$ & 7 & $5,4 \%$ \\
Pâncreas & 3 & $5,3 \%$ & 10 & $7,7 \%$ \\
Vascular & -- & -- & 7 & $5,4 \%$ \\
Bexiga & 2 & $3,7 \%$ & 3 & $2,3 \%$ \\
Vesícula biliar & 1 & $1,7 \%$ & 2 & $1,5 \%$ \\
\hline
\end{tabular}

TABELA 2 - Causas de óbitos dos pacientes que sofreram trauma abdominal e foram submetidos à laparotomias exploradoras. $P<0,05$

\begin{tabular}{lcc}
\hline Causas de óbitos & $\mathbf{n}$ & $\mathbf{\%}$ \\
\hline Choque hipovolêmico & 9 & $47 \%$ \\
Choque séptico & 6 & $32 \%$ \\
Parada cardiorespiratória durante a operação & 3 & $16 \%$ \\
Parada cardiorespiratória durante a extubação & 1 & $5 \%$ \\
\hline Total & $\mathbf{1 9}$ & $\mathbf{1 0 0 \%}$ \\
\hline
\end{tabular}

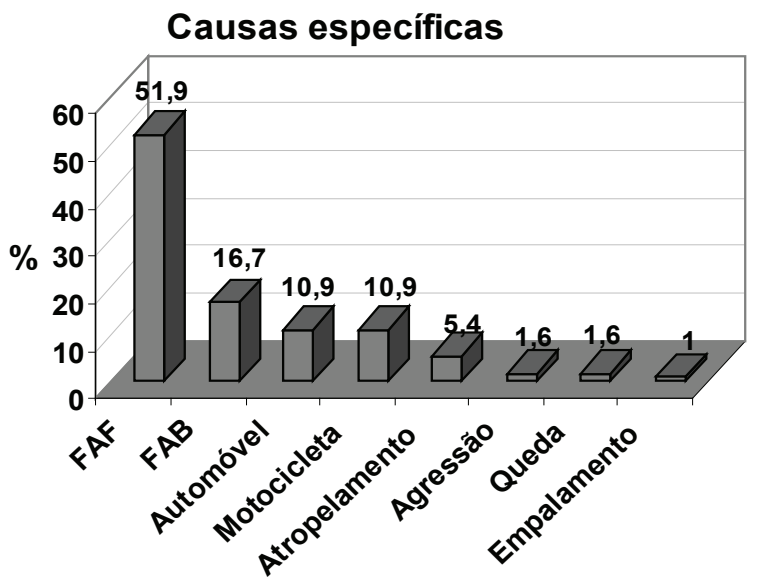

FIGURA 2 - Percentual de causas de trauma abdominal nos pacientes submetidos à laparotomia exploradora. $P<0,05 \mathrm{FAF}=$ ferimento por arma de fogo; FAB $=$ ferimento por arma branca 
No intervalo de 1 a 5 dias, permaneceram internados $41 \%$ (76) dos indivíduos; no intervalo de 6 a 10 dias, esse valor foi de $28,1 \%$ (52) do total.

Na Figura 3, observa-se que 70,3\% (130) dos pacientes receberam alta sem intercorrências e 19,5\% (36) foram submetidos à reintervenção. A mortalidade foi de 10,2\% (19), sendo que 6\% (11) foram a óbito no pós-operatório imediato. Os óbitos que ocorreram no pós-operatório tardio e no período per-operatório representaram $2,1 \%$ (4) cada.

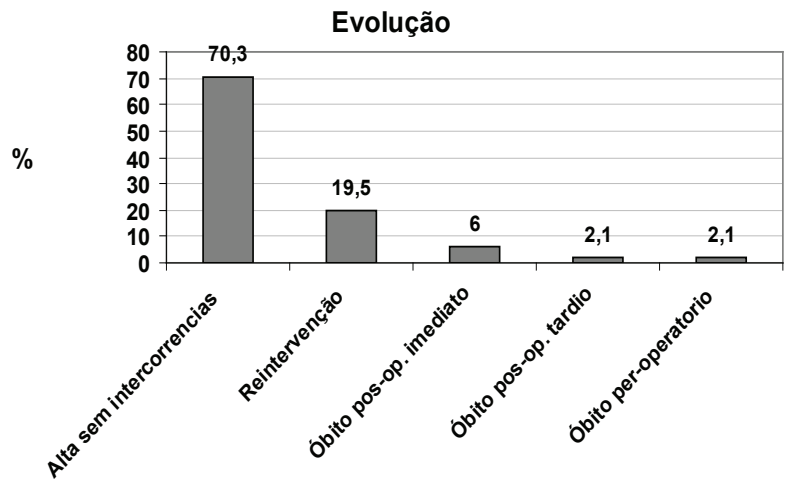

FIGURA 3 - Evolução dos indivíduos que sofreram traumas abdominais e foram submetidos à laparotomia exploradora. $P=0,0001$

Do total de 19 pacientes que foram a óbito, 10 pertenciam ao grupo em que as causas foram o trauma contuso, representando percentual de $17,8 \%$, enquanto que 9 pacientes pertenciam ao grupo em que a causa foi trauma penetrante, representando percentual de $7 \%$.

Dentre as causas de óbitos, $47 \%$ (9) foram devido ao choque hipovolêmico; $32 \%$ (6) devido ao choque séptico (Tabela 2). Observou-se que 47,3\% (9) ocorreram na faixa etária de 20 a 29 anos; nas de 10 a 19 anos e 30 a 39 anos a prevalência foi de $15,8 \%$ cada.

Como traumas associados obteve-se o trauma torácico em 22,2\% (41) do total de indivíduos; fratura de membros em 11,3\% (21); traumatismos cranioencefálicos em 3,8\% (7); fratura de pelve em 2,7\% (5); traumatismo raquimedular em 1\% (2) (Figura 4).

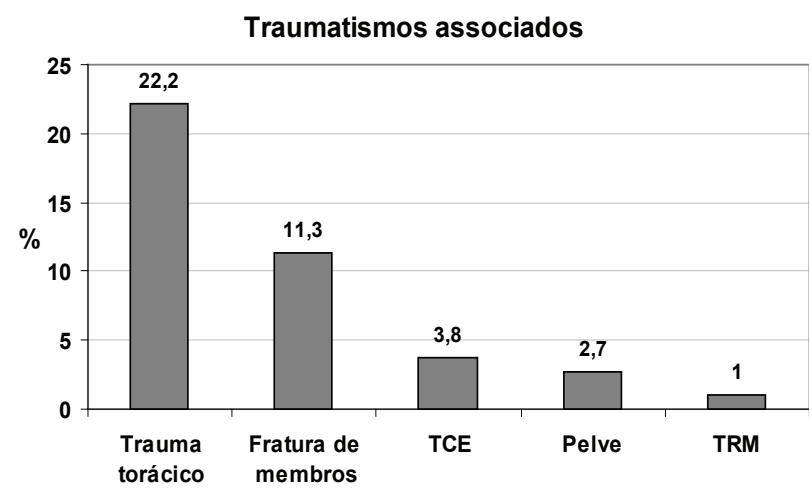

FIGURA 4 - Lesões extra-abdominais associadas ao trauma abdominal de pacientes submetidos à laparotomia exploradora. $P<0,05$ TCE $=$ traumatismo cranioencefálico; TRM = traumatismo raquimedular

\section{DISCUSSÃO}

Constatou-se que o sexo masculino foi o mais afetado, representando $87 \%$, valor muito próximo aos $92 \%$ encontrado no Hospital de Pronto Socorro de Porto Alegre ${ }^{8}$ por Espino et al. ${ }^{9}$. O mesmo padrão de ocorrência também foi verificado por Costa $^{10} \mathrm{em}$ seu estudo, com valor de $89,6 \%$. $\mathrm{O}$ indicador APVP vem sendo apontado como alternativa interessante para comparar diferenças no padrão de mortalidade por sexo. São relevantes as discrepâncias observadas no padrão da mortalidade por sexo em Santa Catarina. Os homens estão mais expostos ao risco de morrer por acidentes, homicídios e suicídios, refletindo funções sociais e riscos diferentes na idade produtiva ${ }^{11}$.

A faixa etária mais acometida foi a terceira década, representando 44,3\%, próxima a de Espino et al. ${ }^{8}$ de 48,8\%. A média de idade no presente estudo foi de 28,6 anos junto com vários estudos que relatam médias de idade na terceira década de vida ${ }^{8,9,10}$. Indivíduos com menos de 15 anos não foram encontrados devido a que o atendimento do trauma infantil não ser realizado no hospital do estudo. De acordo com a Organização Mundial de Saúde, as causas externas são responsáveis por taxas de mortalidade mais elevadas na população jovem, do sexo masculino e que vive em países pobres ${ }^{11}$.

Em relação aos meses do ano, verificou-se que os meses de outubro $(15,6 \%)$ e dezembro (14\%) apresentaram as maiores prevalências de eventos e quando feita a análise trimestral, constatou-se que o $4^{\circ}$ trimestre representou $39,4 \%$ de casos, este valor é superior ao dobro de eventos verificados no $2^{\circ}$ trimestre. Essa diferença pode ser explicada pelo aumento da população na região da grande Florianópolis nos meses de final de ano, devido principalmente, ao turismo.

Verificando as causas específicas, encontrou-se que $69,6 \%$ ocorreram devido a mecanismos penetrantes, sendo o principal deles o ferimento por arma de fogo representando $51,9 \%$ do total de casos. Se comparar com o estudo realizado no Hospital das Clinicas de Botucatu com 200 pacientes, verifica-se que lá os ferimentos penetrantes foram a causa de $54,5 \%$ com o ferimento por arma branca, representando $35 \%$ dos $\operatorname{casos}^{13}$. O ferimento por arma branca também foi verificado por $\operatorname{Costa}^{10}$ como sendo a principal causa do trauma abdominal em $44,5 \%$ vs $29,4 \%$ de arma de fogo.

O estado de Santa Catarina ocupava a $24^{\circ}$ posição no ranking de taxas de homicídios na população total no ano de 1994, e no ano de 2004 a $27^{\circ}$ posição, com taxa de 11,1 homicídios por 100.000 habitantes. Mesmo considerando o impacto das políticas de desarmamento implementadas em 2004 , as taxas de violência homicida continuam elevadas ${ }^{14}$. Apesar de ocupar a última colocação, foi verificado neste estudo o emprego de armas de fogo como principal instrumento no trauma abdominal.

Os mecanismos contusos de trauma representaram $30,4 \%$ do total de indivíduos estudados, o automóvel e a motocicleta apresentaram valores iguais com $10,9 \%$ cada, em discrepância com estudo realizado em Curitiba ${ }^{15} \mathrm{em}$ 
que os mecanismos contusos foram as principais causas em $58,5 \%$. Esperava-se encontrar prevalência maior de traumas contusos, já que o hospital deste estudo localizase próximo à BR-101 e se trata de hospital de referência em trauma.

Observou-se aumento expressivo de casos de traumas devido a mecanismos penetrantes no $4^{\circ}$ trimestre, enquanto o aumento de causas contusas foi menos acentuado, o que leva à sugestão de que nessa época do ano, a probabilidade de ser vítima de arma de fogo é maior.

As laparotomias não-terapêuticas representaram 14\% do total desse procedimento realizados, esse mesmo valor foi encontrado em outro estudo ${ }^{16}$. Em trabalho similar realizado por Meyer ${ }^{17}$ a taxa de laparotomias não-terapêuticas encontradas foi de $11,43 \%$. Ela tende a diminuir com a melhor utilização de meios diagnósticos como a ultra-sonografia e a tomografia computadorizada.

Verificou-se que dentre as estruturas anatômicas afetadas a ordem foi: intestino delgado, fígado e baço. Quando analisadas separadamente, notou-se que o mecanismo contuso lesou principalmente baço, intestino delgado e fígado em consonância com outros estudos $\mathrm{s}^{4,6} \mathrm{em}$ que essas estruturas também foram as mais afetadas. Já o mecanismo penetrante, no presente estudo, lesou principalmente o intestino delgado, o fígado e o intestino grosso; essa mesma seqüência de estruturas afetadas também foi verificada em outros estudos ${ }^{4,6}$.

A taxa de mortalidade global observada foi de $10,2 \%$, sendo este valor superior ao encontrado na literatura revisada, com taxas de $7 \%{ }^{13}$ e $7,24 \%{ }^{9}$. Porém, quando se subdivide a mortalidade por causas de trauma, fica sendo a mais elevada no trauma contuso em relação ao trauma penetrante. As causas determinantes desta diferença são as associações com lesões extra-abdominais (crânio, tórax e esqueleto) e a demora na indicação cirúrgica, fatos observados no trauma fechado ${ }^{3}$.

A principal causa de óbito foi o choque hipovolêmico com $47 \%$, esta associação sendo verificada também por outros autores ${ }^{9,19,20}$.

A principal faixa etária mais acometida de 20 a 29 anos também foi verificada em outros estudos ${ }^{9,21}$. $\mathrm{O}$ adulto jovem apresenta-se como vítima importante, fato que evidencia não só dano social, como também perdas na população economicamente ativa ${ }^{3}$.

São freqüentes os relatos de traumatismos associados ao trauma contuso $0^{4,5,18,22}$, sendo que no presente estudo o principal deles foi o torácico.

A importância do trauma abdominal é que ele constitui uma das principais causas de morte evitáveis no trauma ${ }^{3}$. Os estudos retrospectivos e observacionais são realizados para o levantamento de dados e formulação de hipóteses que poderão ser testada por estudos analíticos, já que são limitados neste ponto. Neste estudo foram apresentadas as estatísticas que podem ser úteis para a manutenção e o planejamento de medidas de prevenção e terapêuticas.

\section{CONCLUSÃO}

O sexo que predominou foi o masculino; a faixa etária predominante foi a terceira década; a causa principal foi o ferimento por arma de fogo; os órgãos mais afetados foram o intestino delgado, fígado e baço; o traumatismo associado ao trauma contuso mais freqüente foi o torácico.

Kruel NF, Oliveira VL, Oliveira VL, Honorato RD, Pinatti BD, Leão FR. Epidemiologic profile of abdominal trauma submitted to laporotomy. ABCD Arq Bras Cir Dig 2007; 20(2):106-10.

ABSTRACT - Background - In Brazil, in the year of 2004, mortality by external causes represented 12,4\% of the total deaths, corresponding to, 124.470 deaths. Abdominal trauma is present in 13\%-15\% of all fatal accidents and, not surprisingly, contributes in a significant way to the late deaths by sepsis. Aim - To describe the epidemiological profile of individuals who suffered abdominal trauma and were submitted to laparotomy treatment, at the HRSJ-HMG, within the period of 2003 to 2006. Methods - The present investigation is constituted of a study with transversal delineation, descriptive and retrospective in 185 individuals, having as a basis the information collecting referring to all the abdominal traumas, submitted to laparotomy treatment, occurred between the periods of 2003 to 2006, based on data from the HRSJ-HMG. Results - The most frequent gender was the male one, which prevailed in $87 \%$ (161), being most of the individuals in the age group of 20 to 29 years old $44,3 \%(82)$ of the total; firearm injury was the main cause of abdominal trauma, representing 51,9\%(96); the most frequent death cause was hypovolemic shock $47 \%$ (6); from the associated extra-abdominal lesions, the one that prevailed was the thoracic trauma with 22,2\% (41). Conclusion - This study showed that the most frequent gender was the male one, the most frequent age group was from 20 to 29 years old, being the main cause firearm injury. The most affected structures were the small intestine, liver and spleen. Most of the patients remained hospitalized within a period of one to five days and are discharged without intercurrences.

HEADINGS - Trauma. Abdomen. Laparotomy.

\section{REFERÊNCIAS}

1 American College of Surgeons. Trauma abdominal. Suporte avançado de vida no trauma para médicos. 7th ed. Chicago; 2004. p.151-68.

2 American College of Surgeons. Avaliação e atendimentos iniciais. Suporte avançado de vida no trauma para médicos. 7th ed. Chicago; 2004. p.13-48.

3 Birolini D. Como anda a epidemia do trauma? Rev Assoc Med Bras. 2001;47:3.

4 Brasil. Ministerio da Saúde. Secretaria executiva. DATASUS. [online] homepage da internet] Sistema de informação sobre mortalidade - SIM. Brasília; 2007. Disponível em http://www.datasus.gov.br

5 Costa OL, Zanon U. Trauma abdominal: gravidade da lesão versus infecção hospitalar. Rev Col Bras Cir. 1991;mar/abr.
6 Edna TH, Bjerkeset T, Myrvoldi HE. Abdominal injuries occurrence and outcome. Tid Sskr Nor Lacgeforen. 1989;109:2111-4.

7 Espino JAR, Dominguez LCA, Barthelemy IR, Hernández IG, Ojeda MJO. Traumas abdominales. Experiência em un servicio cirugia general, 1986 a 1993. Rev Cubana Cir. 2002;2:24-9.

8 Fabian TC, Croce MA. Trauma abdominal, incluindo indicações para laparotomia. In: Mattox KL, Feliciano DV, Moore EE, editores. Trauma. 4th ed. Houston, Tx, McGraw Hil; 2005. p 583-602. 
9 Henry MACA, et al. Trauma abdominal: análise dos aspectos epidemiológicos e clínicos em cidade de médio porte do Estado de São Paulo. Rev Col Bras Cir. 1991;18:143-7.

10 Kruel NF, Araújo PA, Ribeiro ECS. Armadilhas do trauma abdominal. In: Freire E, editor. Trauma a doença do século. São Paulo: Atheneu; 2001. p 2483-90.

11 Meyer L, Kluge, Marusch F, Zippel R, Gastinger I. Der stellenwert der laparoskopie beim abdominaltrauma. Z Chir. 2002;127:533-7.

12 Nakamura N, Yamura A, Shigemori M, Ogawa T, Tokutomi T, Ono J. Final report of de Japan neurotrauma data bank project 1998-2001: 1002 cases of traumatic brain injury. Neurol Med Chir. 2006;46:567-74.

13 Navarro PA, Suarez MA, Bondia JA. Traumatismos abdominales. Nuestra experiências. Rev Cir Esp. 1988;43:45-50.

14 Oliveira BFM et al. Trauma: atendimento pré-hospitalar. Rio de Janeiro: Atheneu, 2001. p.193-8.

15 Peixoto HCG, Souza ML. Anos potenciais de vida pedidos e padrões de mortalidade por sexo em Santa Catarina. Florianópolis, SC.; 1995. Disponível em www.saude.sc.gov.br

16 Pruinelli R, Souza HP, Cardoso AA, Stedile M, Pinto RD. Avaliação do índice de trauma abdominal (ATI): estudo prospectivo de 285 casos. Rev HPS. 1994;40:32-8.
17 Ribas-Filho JM, Malafaia O, Campos ACL, Graumam RQ, Gomes SE, Marochi VL. Prevalência das estruturas atingidas no trauma abdominal. Rev Méd Paraná. 2002.

18 Santa Catarina. Ministério Público de Santa Catarina. Portaria Federal $n^{\circ}$ 737/2001. Política nacional de redução da morbimortalidade por acidentes e violências. Florianópolis; 1996-2007.

19 Souza ER, Lima MLC. Panorama da violência urbana no Brasil e suas capitais. Ciênc Sal Col. 11(2).

20 Tambellini AT, Osanai CH. Epidemiologia do trauma. In: Freire E. Trauma: a doença do século. São Paulo: Atheneu; 2001. p.47-76.

21 Tovar AV, Yepes LET. Trauma abdominal. In: Rodríguez A, Ferrada R, editores. Trauma. Colômbia: Talleres Gráficos de Impresora Ferriva; 1997. p. 307-24.

22 Velmahos, et al. Selective nonoperative management in 1856 patients with abdominal gunshot wounds: should routine laparotomy still be the standard of care? Ann Surg. 2001;234:395-403.

23 Waiselfisz JJ. Homicídios na população total. In: Brasil. Ministério da Saúde. Mapa da violência. Brasília; Gráfica Brasil: 2007. p.20-54.

Conflito de interesse: não há Fonte financiadora: não há Recebido para publicação em: 04/01/2007 Aceito para publicação em: 10/04/2007 